\title{
How specific is synchronous neuronal firing? Wei $\mathrm{Wu}^{* 1}$ and Gordon Pipa ${ }^{2,3}$
}

\author{
Address: ${ }^{1}$ Frankfurt International Graduate School for Science, 60438 Frankfurt, Germany, ${ }^{2}$ Frankfurt Institute for Advanced Studies, 60438 \\ Frankfurt am Main, Germany and ${ }^{3}$ Max-Planck-Institute for Brain Research, 60528 Frankfurt am Main, Germany \\ Email: Wei Wu* - wwu@mpih-frankfurt.mpg.de \\ * Corresponding author
}

from Sixteenth Annual Computational Neuroscience Meeting: CNS*2007

Toronto, Canada. 7-12 July 2007

Published: 6 July 2007

BMC Neuroscience 2007, 8(Suppl 2):P50 doi:I0.I I86/I47|-2202-8-S2-P50

(C) 2007 Wu and Pipa; licensee BioMed Central Ltd.

\section{Background}

Synchronous neuronal firing has been discussed as a potential neuronal code. For testing first, if synchronous firing exists, second if it is modulated by the behaviour, and third if it is not by chance, a large set of tools has been developed. However, to test whether synchronous neuronal firing is really involved in information processing one needs a direct comparison of the amount of synchronous firing for different factors like experimental or behavioural conditions. To this end we present an extended version of a previously published method NeuroXidence [1], which tests, based on a bi- and multivariate test design, whether the amount of synchronous firing above the chance level is different for different factors.

\section{Methods}

In order to make a spike rate correction of an observed amount of joint-spike-event (JSE), we define two time scales: $1 . \tau_{c^{\prime}}$ which defines the fine-temporal cross-structure of interest and is equal to the assumed temporal extension of JSE ( $\sim 5 \mathrm{~ms}), 2 . \tau_{\mathrm{r}^{\prime}}$ which is $\eta$ times slower than $\tau_{\mathrm{c}}$ and equal to a lower bound of rate changes $\left(\tau_{\mathrm{r}}=\eta^{*}\right.$ $\left.\tau_{c^{\prime}} \eta \sim 5\right)$. Using $\tau_{\mathrm{c}}$ and $\tau_{\mathrm{r}}$ the chance amount of JSE is derived based on surrogate data. The latter is generated by random jittering of all spikes in each individual original spike train by an amount smaller than $\tau_{\mathrm{r}}$. Hence, jittering destroys the fine-temporal cross-structure but maintains any other properties of each spike train like the full autostructure and rate co-variation. Next we compute for each trial (m) and each factor (i) the difference between the amount of JSE in the original and jittered spike train $\Delta \mathrm{fm}, \mathrm{i}$.
To assess if $\Delta \mathrm{fm}_{\mathrm{m}, \mathrm{i}}$ is different for different factors, we use a bi-, and multivariate test (Mann-Whitney, Kruskawalis).

\section{Results}

We demonstrate based on toy data that the bi- and multivariate version of NeuroXidence is a conservative and reliable method for detecting modulations in the synchronous firing across different experimental factors. To this end we used various scenarios that had been discussed to induce false positives like rate changes and rate co-variations, low rates and different forms of renewal processes. Furthermore we show results based on simultaneous recordings from awake monkeys performing a short term memory paradigm, that modulations of synchronous firing are correlated to behavior and task conditions.

\section{Acknowledgements}

This work was supported by the Hertie foundation.

\section{References}

I. Pipa G, Riehle A, Grün S: Validation of task-related excess of spike coincidences based on NeuroXidence. Neurocomputing 2007, 70:2064-2068. 